\title{
BMJ Open Association between periodontal disease and osteoporosis in postmenopausal women: a protocol for systematic review and meta-analysis
}

\author{
Jing Qi (D) , ${ }^{1}$ E Liu, ${ }^{1}$ Yu-Feng Guo, ${ }^{1}$ Jie-Mei Hu, ${ }^{2}$ Yu-Ting Liu, ${ }^{3}$ Guang Chen, ${ }^{4}$ \\ Hai-Quan Yue ${ }^{1}$
}

To cite: Qi J, Liu E, Guo Y-F, et al. Association between periodontal disease and osteoporosis in postmenopausal women: a protocol for systematic review and meta-analysis. BMJ Open 2021;11:e049277. doi:10.1136/ bmjopen-2021-049277

\section{- Prepublication history and} additional supplemental material for this paper are available online. To view these files, please visit the journal online (http://dx.doi.org/10.1136/ bmjopen-2021-049277).

$J Q$ and EL are joint first authors.

Received 25 January 2021 Accepted 01 September 2021

Check for updates

(C) Author(s) (or their employer(s)) 2021. Re-use permitted under CC BY-NC. No commercial re-use. See rights and permissions. Published by BMJ.

${ }^{1}$ Stomatology Center of Gansu Provincial Hospital, Gansu Provincial Hospital, Lanzhou, Gansu, China

${ }^{2}$ Bone density test room of Gansu Provincial Hospital, Gansu Provincial Hospital, Lanzhou,

Gansu, China

${ }^{3}$ Radiology of Gansu Provincial Hospital, Gansu Provincial Hospital, Lanzhou, Gansu, China ${ }^{4}$ Department of Stomatology, Affiliated Hospital of Northwest University for Nationalities, Lanzhou, Gansu, China

Correspondence to Dr Hai-Quan Yue; yhq19852020@163.com

\section{ABSTRACT}

Introduction Periodontal disease and osteoporosis are common chronic diseases, especially for the postmenopausal women. Several original studies explore the association, but there still controversial. Therefore, we will conduct this systematic review and meta-analysis to assess the association between periodontal disease and osteoporosis in postmenopausal women.

Methods and analysis This study adheres to the Preferred Reporting Items for Systematic Reviews and Meta-analyses for Protocols. We will systematically search Medline/PubMed, Embase, Cochrane Central Register of Controlled Trials, Web of Science and Scopus from inception to August 2021 to collect all relevant publications, with no restrictions on publication date or languages. Study selection, data extraction and risk of bias assessment will be conducted independently by two trained reviewers independently. The Cochrane's tool for assessing risk of bias, Newcastle-Ottawa Scale and Agency for Healthcare Research and Quality will be used for the risk of bias assessment. OR, HR and risk ratio with $95 \% \mathrm{Cl}$ were considered as the effect size for dichotomous outcomes, weighted mean difference with $95 \% \mathrm{Cl}$ were calculated as the effect size for continuous outcomes. Random-effects models will be used. Heterogeneity between studies will be assessed via the forest plot and $\mathrm{I}^{2}$. Publication bias will detected by funnel plots, Begg's test and Egger's test. The subgroup analyses and sensitivity ananlyses will also be used to explore and interpret the heterogeneity.

Ethics and dissemination This study does not require ethical approval. We will disseminate our findings by publishing results in a peer-reviewed journal.

PROSPERO registration number CRD42021225746.

\section{INTRODUCTION}

Osteoporosis is a systemic skeletal disease and the most prevalent bone disease affecting millions of people worldwide. ${ }^{1}$ It is characterised by a decreased bone mineral density (BMD) and decreased bone microarchitecture, ${ }^{2}{ }^{3}$ and results in an increase in bone fragility and fracture risk. The osteoporotic fractures cause significant mortality rate,
Strengths and limitations of this study

- This study will be the first systematic review and meta-analysis to synthetically investigate the association between periodontal disease and osteoporosis in postmenopausal women.

- This research will be conducted in strict accordance with the relevant methodological guidelines of systematic review and meta-analysis to minimise bias.

- The majority of included studies may be retrospectively designed, which may compromise the results of our study.

- The results of this study may be influent by grey literature bias and heterogeneity in the case definitions of periodontitis.

high medical expenditures and substantial morbidity with low quality of life. ${ }^{4}$ Reducing the growing burden of osteoporosis is one of the aims of Expert Report on diet and chronic disease that WHO/Food and Agriculture Organization (FAO) released. ${ }^{5}$ Osteoporosis affects men and women of all race, especially for the older postmenopausal women, for risk factors differ in age, sex and level of hormones. ${ }^{46}$

Periodontitis is a chronic inflammatory disease that results from the accumulation of dental plaque biofilm, microbial dysbiosis leads to a chronic, non-resolving and destructive inflammatory response, ${ }^{7}$ the tissue destruction that occurs is largely irreversible. ${ }^{8}$ The disease may begin in childhood or adolescence but usually debuts in early adulthood and occasionally in later years. ${ }^{9}$ In 2010 , severe periodontitis was the sixth most prevalent condition and that it affected 743 million $(10.8 \%)$ people aged $15-99$ worldwide. ${ }^{10}$ The global prevalence of severe periodontitis increased steadily until individuals reached approximately 40 years of age and plateaued thereafter. $^{11}$ 
Although periodontal disease is confined locally and osteoporosis is a systemic process, bone loss is characteristic in both case. ${ }^{12}$ Because their main feature influenced by common risk factors, including age, hormonal changes, the presence of cytokines, smoking habit, nutritional intake, educational level and so on. ${ }^{3613}{ }^{14}$ Additionally, postmenopausal women with osteoporosis are susceptible to an excessive response to dental plaque. ${ }^{15}$ Therefore, a hypothesis was proposed that periodontitis is associated with osteoporosis in postmenopausal women, although the biological mechanism underlying this association has not been not fully elucidated. Several original studies explore the association, ${ }^{16}{ }^{17}$ but there still controversial. ${ }^{18}$

High-quality systematic review and meta-anlysis has been regarded as one of the key tools for achieving evidence. The previous systematic review and metaanalysis, published in 2017, explored the association of periodontal attachment loss with low BMD in postmenopausal women. ${ }^{19}$ The meta-analysis focused on the periodontal attachment loss and the clinical attachment level (CAL) is the only outcome. However, loss of alveolar crestal height $(\mathrm{ACH})$, oral hygiene index simplified (OHI-S), probing depth (PD) and percentage of sites with bleeding on probing (BOP) are still the important outcomes for the clinical parameters of periodontal status. In addition, several new evidence published in recent years. ${ }^{180-22}$ Therefore, we will conduct this systematic review and meta-analysis to assess the impact of osteoporosis on the periodontal status of postmenopausal women with periodontal disease.

\section{METHOD}

This protocol followed the Preferred Reporting Items for Systematic Reviews and Meta-analyses (PRISMA) Protocols guidelines. ${ }^{23}$ This systematic review and meta-analysis will be conducted and reported following the reporting guidelines provided in the PRISMA. ${ }^{24}$ No ethical approval and informed consent needed because this is a retrospective study.

\section{Search}

We will systematically search Medline/PubMed, Embase, Cochrane Central Register of Controlled Trials, Web of Science and Scopus from inception to August 2021 to collect publications related to association between periodontal disease and osteoporosis in postmenopausal women. The search strategy will be conducted by medical subject headings with text words. The search terms in each database included ("periodontal diseases" or "parodontosis" or "parodontoses"or "pyorrhea alveolaris") and ("osteoporosis, postmenopausa" or" perimenopausal bone loss*" or "post-menopausal osteoporoses" or "post-menopausal osteoporosis" or "postmenopausal osteoporosis" or "postmenopausal osteoporoses" or "perimenopausal bone losse*”). Online supplemental table S1 describes the detailed full search strategies.
In addition, the reference lists of the included studies and relevant reviews will also be checked, so as to supplement possible relevant literatures. There will no restrictions on publication date or languages. The results of the search will be updated before the final analysis to further identify possible new studies.

\section{Inclusion criteria}

This systematic review and meta-analysis will included the studies evaluated the association between periodontal disease and osteoporosis in postmenopausal women.

\section{Participants}

The participants were postmenopausal women, with no ethnicity, or health status restrictions.

\section{Exposure}

The exposures were participants with osteoporosis and/ or osteopenia.

\section{Comparator}

The comparator was participants with normal BMD.

\section{Study designs}

There are no restrictions on the types of study, and randomised controlled trials(RCTs), cohort studies, case-control studies, longitudinal observational studies, cross-sectional studies and other original studies will be included.

\section{Outcomes}

The outcomes will be clinical parameters of periodontal status, including CAL, PD, gingival recession (GR), percentage of sites with $\mathrm{BOP},{ }^{25}$ the loss of $\mathrm{ACH}$ and $\mathrm{OHI}-$ $\mathrm{S} .{ }^{26} \mathrm{CAL}$ was measured as the distance from the cementenamel junction (CEJ) to the bottom of the pocket ${ }^{2728}$ The $\mathrm{PD}$ reduction, defined as the distance from the gingival margin to the bottom of the pocket. ${ }^{27}{ }^{28} \mathrm{GR}$ was distance from the gingival margin to the CEJ. ${ }^{27}{ }^{28} \mathrm{ACH}$ was the distance from the CEJ to the most coronal point of the alveolar crest immediately adjacent to the root surface, and in the case of a vertical defect, $\mathrm{ACH}$ was the distance from the CEJ to the point immediately adjacent to the root surface at the base of the defect. ${ }^{29}$

\section{Exclusion criteria}

Studies will be excluded if one of the following conditions is met:

1. Qualitative studies, case reports, case series, reviews, letters, comments, notes, animal studies, editorials and conference abstracts.

2. The research data are not complete.

3. The study is not in Chinese or English.

Additionally, studies with the same sample, we will select the study with the longest follow-up time and the largest sample size.

\section{Study selection}

Two trained reviewers will independently select the included studies based on the eligibility criteria. The 
Rayyan web (https://rayyanai/) will be applied. The titles and abstracts of the citations retrieved by the literature search will screened for potential studies. The full text is then screened according to eligibility criteria. Any disagreement will be resolved through discussions. The selection process will be recorded in a PRISMA flow chart.

\section{Data extraction}

A standard form will be used to extract data from the included studies. Two trained reviewers will perform the data extraction independently. Discrepancies between the reviewers will be resolved either by discussion or by a third reviewer. To identify other relevant study data, we will contact the authors of published studies for incomplete data.

Information to be extracted include four domains: (1) study characteristics (first authors, year of publication, country of participants, duration of study, number of participants); (2) participants information (age, ethnicity, the status of periodontal disease); (3) methods (study design, measures of exposure, outcomes and the criteria of periodontal disease and osteoporosis) and (4) outcomes (clinical parameters of periodontal status, HR, OR, risk ratio (RR), 95\% CI, mean and $\mathrm{SD}$ ).

\section{Risk of bias assessment}

Two researchers will independently assess the risk of bias (RoB) of all included studies. Any question will be solved through discussion or by a third reviewer. The Cochrane's tool for assessing $\operatorname{RoB}^{30}$ will be used to evaluate the RoB for RCTs. The RoB tool covers six domains of bias: selection bias, performance bias, detection bias, attrition bias, reporting bias and other bias. The Newcastle-Ottawa Scale will be used to assess the RoB for cohort studies and case-control studies. ${ }^{31}$ It comprises eight questions grouped under three broad dimensions: selection of groups under study, comparability of groups under study, and outcome ascertainment. The scale is given a score of $0-9$ based on selection, comparability and outcome. We will represent 'low', 'medium' and 'high' quality research with scores of 0-3, 4-6and 7-9, respectively. An 11-item checklist is recommended by Agency for Healthcare Research and Quality (available from: https://www. ncbi.nlm.nih.gov/books/NBK35156/) to evaluated the cross-sectional studies. The quality is assessed as 'low', 'medium' and 'high' quality with scores of $0-3,4-7$ and $8-11$, respectively.

\section{Data synthesis and statistical analysis}

The available evidence will be synthesised in a metaanalysis on the basis of enough relevant studies. Statistical analysis was performed with STATA software, V.15.0 (StataCorp).

In this systematic review and meta-analysis, HR, OR or RR with $95 \%$ CI were considered as the effect size for dichotomous outcomes, weighted mean differences with 95\% CI were calculated as the effect size for continuous outcomes.
Forest plots were produced to visually assess the effect size and corresponding 95\% CI using random-effects models of DerSimonian and Laird. Heterogeneity between studies will be assessed via the forest plot and the Cochran $Q$ test $(p<0.05$ considered statistically significant), while $\mathrm{I}^{2}$ values described the total variation between studies. $\mathrm{I}^{2}$ values of $<25 \%, 25 \%-50 \%$ and $>50 \%$ indicated low, moderate and high heterogeneity, respectively.

\section{Publication bias}

We will assess the publication bias of included studies when there are at least 10 studies reporting the primary outcomes. Visual inspection of funnel plots, and statistical assessments using the Begg-Mazumdar rank correlation $^{32}$ and Egger's regression test ${ }^{33}$ will be used to detect publication bias.

\section{Additional analysis}

Subgroup analyses will be used to explore and interpret the sources of heterogeneity, and to evaluate whether there are different. If data available, the subgroup analyses will be based on (1) study designs, (2) CAL $\geq 4 \mathrm{~mm}$ and $\geq 6 \mathrm{~mm}$, (3) definitions of periodontitis, (4) the BMD evaluations (dual energy X-ray absorptiometry (DXA) of lumbar spine, DXA of proximal femur, DXA of forearm, ultrasound and lateral radiographs) and (5) other characteristics of participants and exposure, which have influence on the periodontal disease and periodontal disease for postmenopausal women.

We will adopt sensitivity analyses to explore the sources of high heterogeneity and to evaluate the stability of the results by excluding studies with high/medium RoB or other special characteristics. We will also conduct sensitivity analyses by serially excluding one study at a time to determine the influence of individual studies on the overall estimates and to explore whether the results were robust.

\section{Patient and public involvement}

Patients and/or the public involved.

\section{Current study status}

Preliminary searches.

\section{ETHICS AND DISSEMINATION}

This systematic review and meta-analysis involves no patient contact and no interaction with healthcare providers or systems. We will disseminate the findings of this study through the presentation at scientific publication in a peer-reviewed journal.

\section{DISCUSSION}

Periodontal disease and osteoporosis are common chronic diseases, especially for the women in postmenopause. A hypothesis was proposed that periodontitis is associated with periodontitis in postmenopausal women, because bone loss is their common characteristic and 
their main feature influenced by common risk factors (eg, age, hormonal changes, etc). Therefore, we will conduct this systematic review and meta-analysis, which is the first to synthetically investigate the association between periodontal disease and osteoporosis in postmenopausal women with more comprehensive outcomes. The findings may fill the gap in this field.

There may be several limitations for this systematic review and meta-analysis. First, the quality of included studies have impact on the quality of evidence for this meta-analysis, and the quality of evidence for this metaanalysis may not high for lack of prospective original studies, although these retrospective studies presented good scientific qualities. Second, case definitions of periodontitis maybe different, ${ }^{18}$ which may result in high heterogeneity. Third, the measurements of ACH, OHI-S, PD and BOP are dependent, to a large extent, on the skills of the dental examiners, which may cause high heterogeneity. ${ }^{34}$ The results may be influent by grey literature bias, since no additional searches are performed for unpublished studies and literature in this study.

The results of this study hope to provide high-quality evidence for the related research. And the final report of this systematic review and meta-analysis in the form of scientific paper will be published in peer-reviewed journals.

Contributors $\mathrm{H}-\mathrm{QY}$ and $\mathrm{JQ}$ conceived the idea and design the protocol. H-QY, JQ, EL and Y-FG drafted the manuscript. H-QY, JQ, EL, Y-FG, J-MH, Y-TL and GC critically revised the manuscript for methodology and intellectual content. H-QY, JQ and $E L$ are the guarantors of the review. All authors approved the final version of this manuscript.

Funding This work was supported by Gansu Provincial Hospital, grant number: GSWSKY2020-77 and 18GSSY1-7.

Competing interests None declared.

Patient consent for publication Not required.

Provenance and peer review Not commissioned; externally peer reviewed.

Supplemental material This content has been supplied by the author(s). It has not been vetted by BMJ Publishing Group Limited (BMJ) and may not have been peer-reviewed. Any opinions or recommendations discussed are solely those of the author(s) and are not endorsed by BMJ. BMJ disclaims all liability and responsibility arising from any reliance placed on the content. Where the content includes any translated material, BMJ does not warrant the accuracy and reliability of the translations (including but not limited to local regulations, clinical guidelines, terminology, drug names and drug dosages), and is not responsible for any error and/or omissions arising from translation and adaptation or otherwise.

Open access This is an open access article distributed in accordance with the Creative Commons Attribution Non Commercial (CC BY-NC 4.0) license, which permits others to distribute, remix, adapt, build upon this work non-commercially, and license their derivative works on different terms, provided the original work is properly cited, appropriate credit is given, any changes made indicated, and the use is non-commercial. See: http://creativecommons.org/licenses/by-nc/4.0/.

ORCID iD

Jing Qi http://orcid.org/0000-0002-0591-0412

\section{REFERENCES}

1 Pavone V, Testa G, Giardina SMC, et al. Pharmacological therapy of osteoporosis: a systematic current review of literature. Front Pharmacol 2017;8:803.

2 Lau RY-C, Guo X. A review on current osteoporosis research: with special focus on disuse bone loss. J Osteoporos 2011;2011:293808.
3 Pouresmaeili F, Kamalidehghan B, Kamarehei M, et al. A comprehensive overview on osteoporosis and its risk factors. Ther Clin Risk Manag 2018;14:2029-49.

4 Christodoulou C, Cooper C. What is osteoporosis? Postgrad Med J 2003;79:133-8.

5 World Health Organization. WHO/FAO release independent expert report on diet and chronic disease. World Health Organization, 2020.

6 Ayed MS, Alsharif AF, Divakar DD, et al. Evaluating the possible association between systemic osteoporosis and periodontal disease progression in postmenopausal women. Dis Mon 2019;65:193-215.

7 Preshaw PM, Bissett SM. Periodontitis and diabetes. Br Dent $J$ 2019;227:577-84.

8 Winning L, Polyzois I, Sanmartin Berglund J, et al. Periodontitis and airflow limitation in older Swedish individuals. J Clin Periodontol 2020;47:715-25.

9 Slots J. Periodontitis: facts, fallacies and the future. Periodontol 2000 2017;75:7-23.

10 Frencken JE, Sharma P, Stenhouse L, et al. Global epidemiology of dental caries and severe periodontitis - a comprehensive review. $J$ Clin Periodontol 2017;44:S94-105.

11 Papapanou PN, Susin C. Periodontitis epidemiology: is periodontitis under-recognized, over-diagnosed, or both? Periodontol 2000 2017;75:45-51.

12 Holmstrup P, Damgaard C, Olsen I, et al. Comorbidity of periodontal disease: two sides of the same coin? an introduction for the clinician. J Oral Microbiol 2017;9:1332710.

13 Lauritano D, Moreo G, Carinci F, et al. Aging and oral care: an observational study of characteristics and prevalence of oral diseases in an Italian cohort. Int J Environ Res Public Health 2019:16:3763.

14 Contaldo M, Itro A, Lajolo C, et al. Overview on osteoporosis, periodontitis and oral dysbiosis: the emerging role of oral microbiota. Appl Sci 2020;10:6000.

15 Brennan RM, Genco RJ, Hovey KM, et al. Clinical attachment loss, systemic bone density, and subgingival calculus in postmenopausal women. J Periodontol 2007;78:2104-11.

16 Al Habashneh R, Alchalabi Haifa'a, Khader YS, et al. Association between periodontal disease and osteoporosis in postmenopausal women in Jordan. J Periodontol 2010;81:1613-21.

17 Singh A, Sharma RK, Siwach RC, et al. Association of bone mineral density with periodontal status in postmenopausal women. $J$ Investig Clin Dent 2014;5:275-82.

18 Gil-Montoya JA, Garrido-Martínez M, Barrios-Rodríguez R, et al. Association between low bone mineral density and periodontitis in generally healthy perimenopausal women. $J$ Periodontol 2021;92:95-103.

19 Penoni DC, Fidalgo TKS, Torres SR, et al. Bone density and clinical periodontal attachment in postmenopausal women: a systematic review and meta-analysis. J Dent Res 2017;96:261-9.

20 Sooragonda B, Cherian KE, Jebasingh FK, et al. Longitudinal changes in bone mineral density and trabecular bone score following yearly zoledronic acid infusion in postmenopausal osteoporosis-a retrospective-prospective study from southern India. Arch Osteoporos 2019;14:79.

$21 \mathrm{Zhu} J$, Li JH, Yuan TT, et al. [Relationship between periodontitis and osteoporosis in postmenopausal women]. Beijing Da Xue Xue Bao Yi Xue Ban 2019;51:1115-8.

$22 \mathrm{Zhu}$ J, Li JH, Yuan TT, et al. [Association between osteoporosis and severe periodontal attachment loss in postmenopausal women]. Zhonghua Kou Qiang Yi Xue Za Zhi 2020;55:159-64.

23 Shamseer L, Moher D, Clarke M, et al. Preferred reporting items for systematic review and meta-analysis protocols (PRISMA-P) 2015: elaboration and explanation. BMJ 2015;350:g7647.

24 Page MJ, McKenzie JE, Bossuyt PM, et al. The PRISMA 2020 statement: an updated guideline for reporting systematic reviews. BMJ 2021;372:n71.

25 Lang NP, Joss A, Orsanic T, et al. Bleeding on probing. A predictor for the progression of periodontal disease? J Clin Periodontol 1986;13:590-6.

26 Greene JC, Vermillion JR. The simplified oral hygiene index. J Am Dent Assoc 1964;68:7-13.

27 Iorio-Siciliano V, Ramaglia L, Isola G, et al. Changes in clinical parameters following adjunctive local sodium hypochlorite gel in minimally invasive nonsurgical therapy (MINST) of periodontal pockets: a 6-month randomized controlled clinical trial. Clin Oral Investig 2021;25:5331-40.

28 De Ry SP, Roccuzzo A, Lang NP. Long-term clinical outcomes of periodontal regeneration with enamel matrix derivative (EMD): a retrospective cohort study with a mean follow-up of 10 years. $J$ Periodontol 2021 doi:10.1002/JPER.21-0347 
29 Wactawski-Wende J, Hausmann E, Hovey K, et al. The association between osteoporosis and alveolar Crestal height in postmenopausal women. J Periodontol 2005;76:2116-24.

30 Higgins JPT AD, Sterne J. Cochrane Handbook for Systematic Reviews of Interventions Version 5.1.0 [updated March 2011]. London: The Cochrane Collaboration, 2011. www.cochranehandbook.org

31 Wells BS, D GA, O'Connell JP, Welch V, et al. The NewcastleOttawa scale (NOS) for assessing the quality of nonrandomised studies in meta-analyses, 2019. Available: http://www.ohri.ca/ programs/clinical_epidemiology/oxford.asp [Accessed 23 Oct 2020].

32 Begg CB, Berlin JA. Publication bias: a problem in interpreting medical data. J R Stat Soc Ser A Stat Soc 1988;151:419-63.

33 Egger M, Davey Smith G, Schneider M, et al. Bias in meta-analysis detected by a simple, graphical test. BMJ 1997;315:629-34.

34 Hefti AF, Preshaw PM. Examiner alignment and assessment in clinical periodontal research. Periodontol 2000 2012;59:41-60. 